\title{
Characterization of «solar» multicrystalline silicon by local measurements
}

\author{
V.G. Popov \\ Institute of Semiconductor Physics of NASU, 45, prospect Nauki, 03028, Kyiv, Ukraine
}

\begin{abstract}
The features of local measurements of «solar» multicrystalline silicon (mc-Si) parameters are surveyed using examples of grain sizes, diffusion length of minority non-equilibrium charge carriers $L_{d}$ and effective reflectivity of light $R$. It is revealed that the crystal grains in mc-Si have 4 groups of the reference sizes. The errors of the single local measurements of parameters are spotted. It is shown that the values of explored parameters are distributed under the normal law (the Gauss function). The algorithm to obtain the average values of mc-Si parameters with given precision is described. The used experimental procedures for the express non-destructive check of $L_{d}$ and $R$ in the mc-Si samples are briefly considered.
\end{abstract}

Keywords: multicrystalline silicon, solar cell, local measurements, errors, statistical distributions.

Paper received 21.03.01; revised manuscript received 04.04.01; accepted for publication 13.07.01.

\section{Introduction}

Coarse-grained multicrystalline silicon (mc-Si) is widely used now for manufacturing the solar energy photovoltaic converters - solar cells (SC) and modules. About $40 \%$ of the crystalline silicon-based SC production is carried out using mc-Si grown as ingots by a method of a directional crystallization [1]. Mc-Si cost is lower than that of the monocrystalline silicon, and this fact is the strong stimulating factor for the works in this direction.

At development of the SC design and technologies the set of the characteristics both for initial silicon and material after particular technological operations is necessary to know. Mc-Si, contrary to monocrystalline one, is essentially non-uniform material, therefore its parameters cannot be correctly spotted from the single measurements in local areas of a chip. Such measurements are usually used in the basic procedures of the check specific $(\rho)$ and surface resistance, diffusion length of minority non-equilibrium charge carriers $\left(L_{d}\right)$, reflectivity $R$ of Si and structures with anti-reflecting coatings. The fact of presence of $\rho, L_{d}$ non-uniform planar distributions in mc-Si and their influence to the SC parameters is confirmed experimentally, in particular, in $[2,3]$.

It is known that characterization of non-uniform objects by measurements on their local sites, for deriving the parameter average values and determining error val- ues, requires to use a large number of measurements and handling the obtained results by methods of a mathematical statistics.

In this paper parameters of a statistical distribution of grain sizes as well as $L_{d}$ and $R$ values in the samples of «solar» mc-Si are considered, and the algorithms of realization of local measurements of these parameters for deriving their values characterizing mean magnitudes with the desirable precision for concrete amount of measurements are described. The used experimental procedures are briefly surveyed.

\section{Experiment}

P-type boron doped mc-Si samples with a mean specific resistance of $1.4 \mathrm{Ohm} \cdot \mathrm{cm}$ were investigated. Samples had the shape of wafers cut perpendicularly to the crystallization direction. Sample surfaces were not polished, and for removal of a dislocated layer were etched in a CPtype etchant by a depth of 5-10 $\mu \mathrm{m}$.

The $L_{d}$ parameter was measured by a method of spectral dependencies of a surface photo-voltage $V_{p h}$, for the first time described in [4]. The possible sources of errors marked in [5] were taken into account when calculating $L_{d}$ from the data of measurements. In particular, the sample thickness should be not less, than 3 times more, than the inverse absorption constant $\alpha$ of a sounding light, 


\section{V.G. Popov: Characterization of the «solar» multicrystalline silicon by ...}

and not less, than 2 times more than $L_{d}$ value; thickness of the surface space charge region in a sample should be much less, than $L_{d}$. In the number of cases correct $L_{d}$ measurements can be carried out at infringement of these requirements (see, for example [5, 6]).

A schematic view of experimental setup for $V_{p h}$ measuring and design of a sample holder are given in Fig. 1. The set up allows to measure $L_{d}$ value in any site of a sample of a diameter up to $300 \mathrm{~mm}$ and thickness up to 25 $\mathrm{mm}$, and also on samples with non-parallel plate sides. Site localization of the $V_{p h}$ measurements was given by the field electrode area $\left(5 \times 25 \mathrm{~mm}^{2}\right)$, and by the illumination area (that was varied from $4 \times 15 \mathrm{~mm}^{2}$ to $1 \times 4 \mathrm{~mm}^{2}$ ). Measurements were carried out at the arbitrary sites of the sample surface.

The dependence of $V_{p h}$ amplitude on $\alpha$ in the fundamental absorption range for mono- and mc-Si can be described in most of cases by the next simplified formula

$$
V_{p h}=V_{p h m} \alpha L_{d}\left(1+\alpha L_{d}\right)^{-1}
$$

where $V_{p h m}=V_{p h}$ at the near-surface light absorption $\left(\alpha L_{d}>>1\right)$.

When processing the obtained experimental data, the $V_{p h}$ signal should be normalized to the amount of absorbed light quanta for each wavelength. Relevant normalization function can be obtained using non-selective to light wavelength (bolometer, thermoelectric calorimeter) or gauged photo-detector.

To be convinced of a correctness when determining $L_{d}$, this parameter was also measured on samples made of monocrystalline $\mathrm{Si}$ by a method of kinetic dependencies of photoconductivity relaxation and method of coordinate dependencies of photocurrent at point Schottky contact and scanning light probe along the sample surface. The results obtained by all three methods coincided.

Parameter $R$ was determined at the wavelength of GaAs light-emitting diode radiation $(920 \ldots 935 \mathrm{~nm})$ with application of opto-pair with the unclosed optical channel. Tested area was about $20 \mathrm{~mm}^{2}$. In the case of $\mathrm{mc}-\mathrm{Si}$, having rough surface, $R$ represents an effective param-

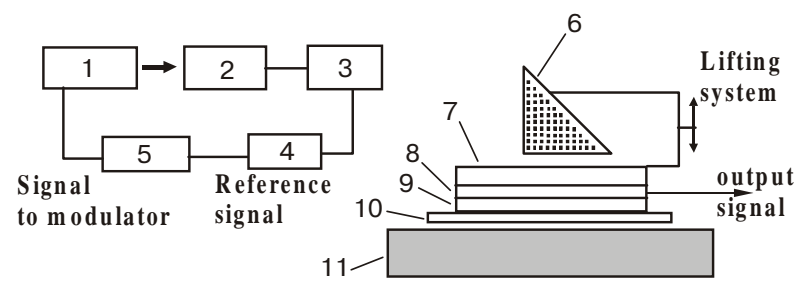

Fig. 1. The simplified scheme of the setup for a surface photovoltage measurements and design of a sample holder. 1 - source of modulated monochromatic light with a tunable wavelength, 2 - sample in a holder, 3 - pre-amplifier with a high input impedance, 4 - lock-in amplifier, 5 - generator, 6 - prism, 7 - glass plate, 8 - ITO layer, 9 - $\mathrm{TiO}_{2}$ layer, 10 - sample, $11-3 \mathrm{D}$ coordinated and angle-adjusted table.

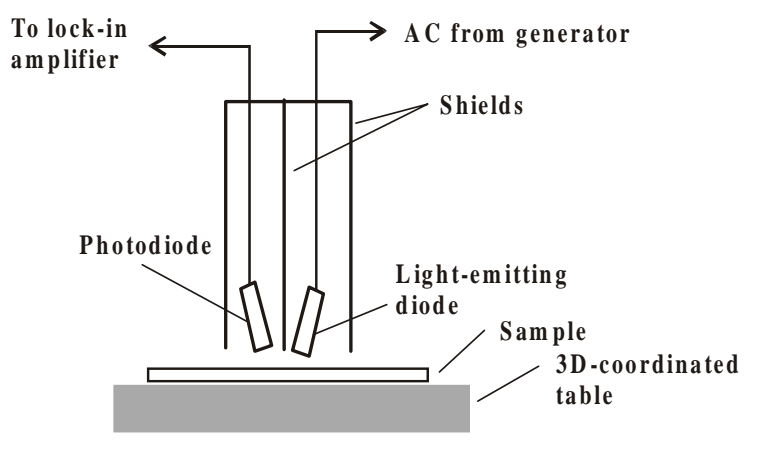

Fig. 2. The scheme of the setup for reflection measurements.

eter including both reflection and light scattering on inhomogeneities of a sample surface. The simplified blockscheme of the setup for the $\mathrm{R}$ parameter measuring and construction of the optoelectronic cell are represented in Fig. 2. The R parameter has been measured at arbitrary sites of the sample surface by scanning the 3D-coordinated table with a sample.

As our experiments have shown, depending on the test geometry (distance of the optoelectronic cell from the sample surface, the angle of its inclination), and on the character of Si surface roughness, the light intensity, registered by the photodiode, may be both some higher and some lower than for the sample with polished surface.

Distribution of grains by sizes on the sample surface was investigated by optical microscopy.

\section{Results and discussion}

In Fig. 3a, the histogram of typical distribution of grains by the mean size $d$, constructed by results of 100 measurements on arbitrary sites of a sample surface is given. It is obvious that one can separate 4 groups of grains: small $\left(d_{m 1}=0.15 \mathrm{~mm}\right)$, medium $\left(d_{m 2}=2.9 \mathrm{~mm}\right)$, large $\left(d_{m 3}=8.2 \mathrm{~mm}\right)$, and extra large $\left(d_{m 4}=15 \mathrm{~mm}\right)$. The distributions of the first three groups of grains are approximated by the sum of three Gauss functions $Y_{i}$ (curve in Fig. 3a):

$Y_{i}=\left[1 / \sigma_{i}(\sqrt{2} \pi)\right] \exp \left[-\left(x-x_{m i}\right)^{2} / 2 \sigma_{i}^{2}\right]$

where $i=1,2,3$.

It is reasonable to believe that the given distribution does not reflect the fact, that exactly large and extra large grains, though their concentration is small, occupy the main area on a sample surface and, thus, mainly determine its characteristics, see Fig. 3b. On the other hand, the concentration of small grains determines basically the area of inter-grain boundaries, the part from which can have recombination and electrical activity and, thus, influence on solar cell parameters [2].

The histogram of distribution of the $L_{d}$ parameter values for a mc-Si sample obtained by 100 measurements on arbitrary sites of a surface (points) is given in Fig. 4a. 
V.G. Popov: Characterization of «solar» multicrystalline silicon by ...
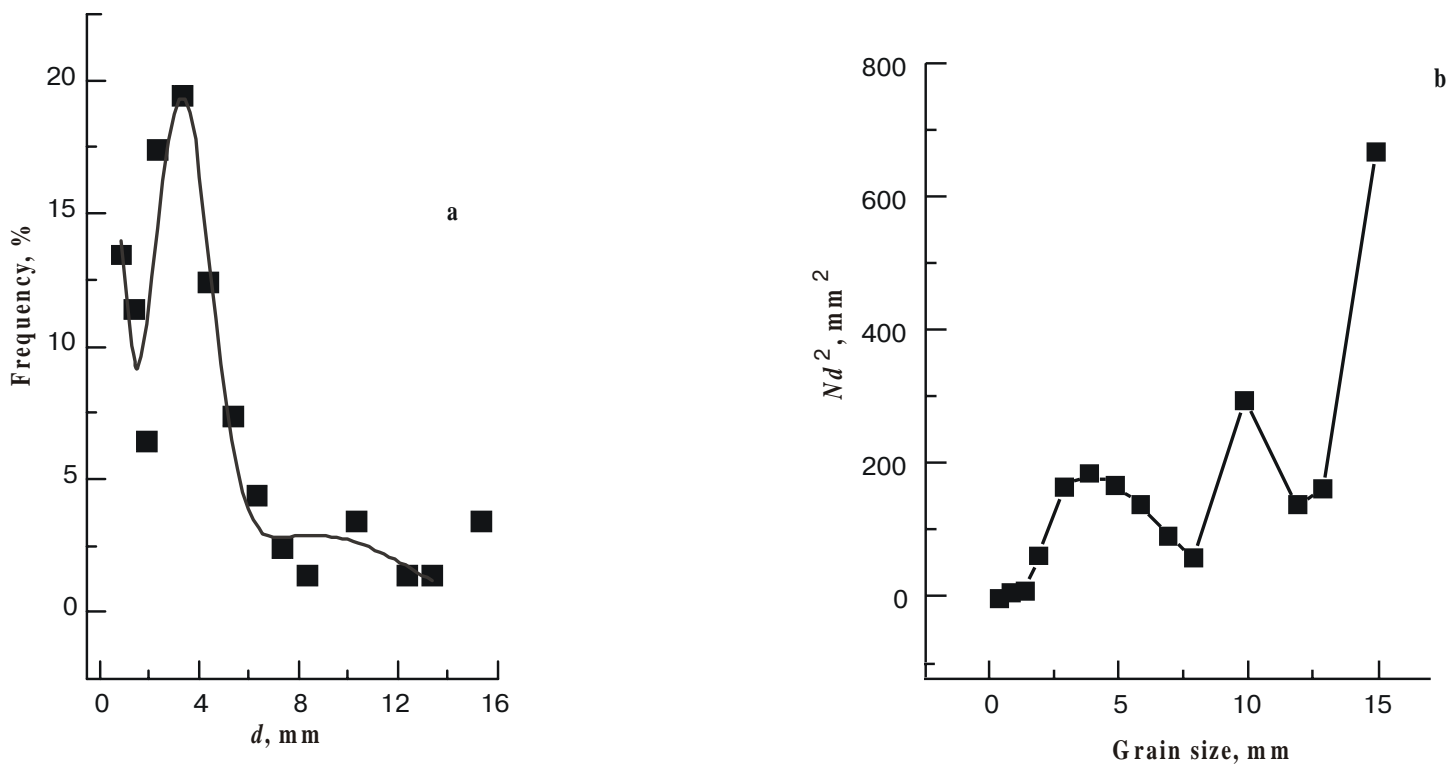

Fig. 3. The histogram of a distribution of grains in a mc-Si sample by sizes. Points - experiment, curve - approximation by the sum of three Gauss functions (a), and dependence of an area, occupied on the mc-Si wafer (see Fig. 3a) by grains of different sizes (approximation by square grains) on grain size (b).

For comparison in Fig. $4 \mathrm{~b}$ the similar histogram for a monocrystalline silicon sample obtained at the same conditions is shown. These measurements were carried out for the illuminated area of $1 \times 15 \mathrm{~mm}^{2}$.
The data of Fig. $4 \mathrm{~b}$ can characterize precision of the used experimental setup. Really, the obtained distribution is approximated by the Gauss function (full curve in Fig. 4b). For the Fig. 4b, data the width parameter of a

a)
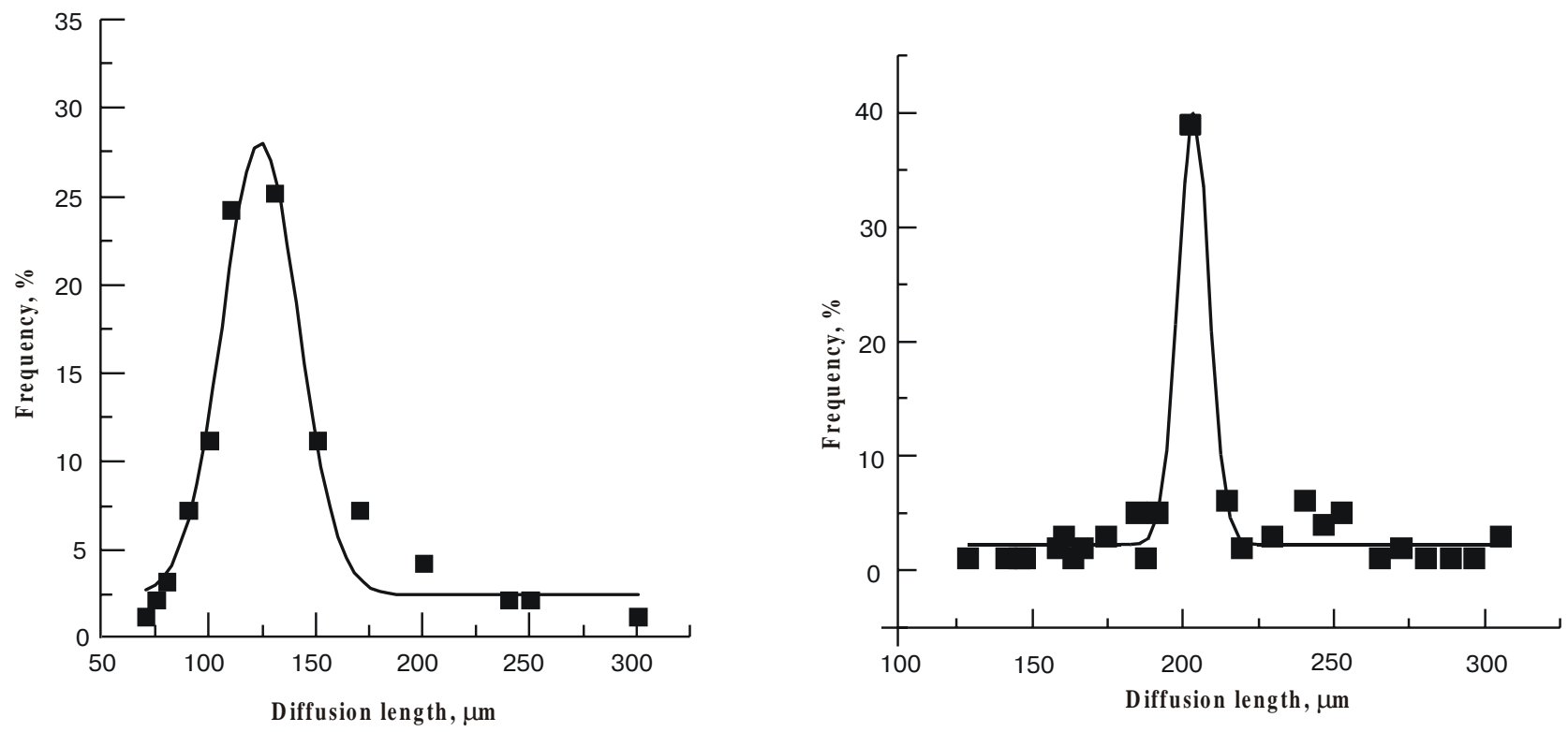

Fig. 4. Histograms of $L_{d}$ value distributions for mc-Si (a) and monocrystalline Si (b) samples. Points - experiment, curves - approximation by the Gauss functions. 
normal distribution is $2 \sigma=10.271 \mu \mathrm{m}$ at the mean $L_{d}$ value of $200.93 \mu \mathrm{m}$. From this, according to [7], the standard deviation $(\sigma=5.13 \mu \mathrm{m})$, arithmetical mean error $(r=$ $0.8 \sigma=4.11 \mu \mathrm{m})$ and relative error $(2.04 \%)$ can be determined. It is necessary to underline that data of Fig. $4 \mathrm{~b}$ are obtained on a sample of monocrystalline silicon not subjected to any thermal and other treatments, which, according to [8], can increment planar inhomogeneity of $L_{d}$ and other parameters.

For the Fig. $4 \mathrm{a}$, data the width parameter is $35.71 \mu \mathrm{m}$ at mean $L_{d}=123.40 \mu \mathrm{m}$. Accordingly, the standard deviation is equal to $17.85 \mu \mathrm{m}$, arithmetical mean error $14.28 \mu \mathrm{m}$, and the relative error $-11.6 \%$.

Thus, the histogram shown in Fig. 4 a contains the information on a statistical distribution of $L_{d}$ values for a concrete mc-Si sample. However, for its deriving, the considerable amount of measurements (by our data - not less than $80 \ldots 90$ ) is necessary, which essentially reduces productivity of operation, especially at lack of computer system for measurements and data processing. For diminution of amount of measurements, an ideal variant would be diminution of their locality (output of a signal from the all sample at irradiating of all its surface), as it is made at measurements of the parameters of final SCs. However, for the majority of experimental techniques this approach, owing to the different reasons, is inapplicable. Further, we have carried out the examinations of the $L_{d}$ distribution as at larger $\left(4 \times 15 \mathrm{~mm}^{2}\right)$, and smaller $\left(1 \times 4 \mathrm{~mm}^{2}\right)$ areas of an illuminated surface, at the invariable area of a field electrode. It has found that the $L_{d}$ distribution practically does not vary. The main reason of this, in our opinion, is influence of a surface space charge region on a planar spreading of non-equilibrium charge carriers. Influence of light scattering on a rough surface of a sample and multiple reflection echoes in a «sample - field electrode» system is also possible.

It is known [9] that if the distribution of values of a physical parameter is featured by the Gauss function, the probability that, at the single measuring, the result obtained will differ from its mean value no more, than by the standard deviation, makes about 0.68 . With the probability of 0.32 results (for concrete data of Fig. 4b) can lay in the range from 70 up to $300 \mu \mathrm{m}$, i.e. does not content almost any useful information. Even more errors arise if it is necessary to check $L_{d}$ changes in the samples after different technological treatments (gettering, annealings etc.), as if the required value is equal to a difference of two quantities, its dispersion $\sigma^{2}$ is equal to the sum of dispersions for each of these quantities [7]. For the Fig. 4a data, for example, the mean standard deviation of single measurements of a difference of $L_{d}$ values reaches $\pm 25 \mu \mathrm{m}$. It is clear that all $L_{d}$ changes smaller than this error at the single measurements can not be correctly captured at all.

The indicated difficulties can be overcome using magnification of the amount of measurements. If the parameter distribution is featured by the Gauss function, as it takes place in all investigated mc-Si samples, according to [9] the mean error of $N$ measurements $\Delta x_{N m}$ diminishes under the law
$\Delta x_{N m}=\Delta x_{m} / \sqrt{N}$

where $\Delta x_{m}$ is the error of the single measurement.

Thus, making up the desirable quantity of a mean error for $L_{d}$ (or for the difference of $L_{d}$ values for an initial sample and the sample after any technological treatment) and beforehand knowing the parameters of a typical statistical $L_{d}$ distribution for the samples of the given batch, it is easy to determine the necessary amount of measurements. By our data, for determination of $L_{d}$ with the precision of $15 \ldots 20 \%$, it is enough to perform from 5 to 10 measurements. For a sample with the mean $L_{d}$ value of $123 \mu \mathrm{m}$ (approximation by the Gauss function in the result of 100 measurements) the results of averaging for the different amount of measurements are given in Table 1.

One have to bear in mind that the errors calculated from the results of few series of the identical local measurements of non-uniform sample are not the same, but, like measured parameter itself, have some statistical distribution of values.

It is interesting that the mean $L_{d}$ values, given in Table 1, are always some higher, than the $L_{d m}$ value obtained as a maximum of the Gauss fit (Fig. 4a). The $\Delta L_{d}$ values also are some higher that that calculated using formula (3). These discrepancies are connected with the contribution of the extra large mc-Si grains to the statistical distribution of $L_{d}$ values [2]. This effect shows itself by spreading the distribution to higher $L_{d}$ values, Fig. 4a.

In Fig. 5 the histogram of a statistical distribution of the parameter $R$ values for a mc-Si sample is given. Also shown are the $R$ values (for the some concrete geometry of the experiment) for a reflection from surfaces of monocrystalline Si wafer. As the reflectivity of Si does not depend on a surface crystallographic orientation, any $R$ difference from its value for a single-crystal polished surface is connected with additional light scattering on surface roughness. It is confirmed by the comparison of the $R$ values for polished and ground surfaces of a monocrystalline sample (Si wafer with the standard treatment of surfaces used in microelectronics production), see Fig. 5.

Contrary to a single crystal, the $R$ parameter for mcSi has straggling values at measurements in different sites

Table 1. The mean $L_{d}$ and $\Delta L_{d}$ values in their dependence on number of local measurements of the mc-Si sample.

\begin{tabular}{ccc}
\hline $\begin{array}{c}\text { Number of } \\
\text { measurements }\end{array}$ & $L_{d}, \mu \mathrm{m}$ & $\Delta L_{d}, \mu \mathrm{m}$ \\
\hline 5 & 142 & 19 \\
\hline 10 & 138 & 15 \\
\hline 25 & 126 & 3 \\
\hline 50 & 129 & 6 \\
\hline 75 & 128 & 5 \\
\hline 100 & 127 & 4 \\
\hline \hline
\end{tabular}




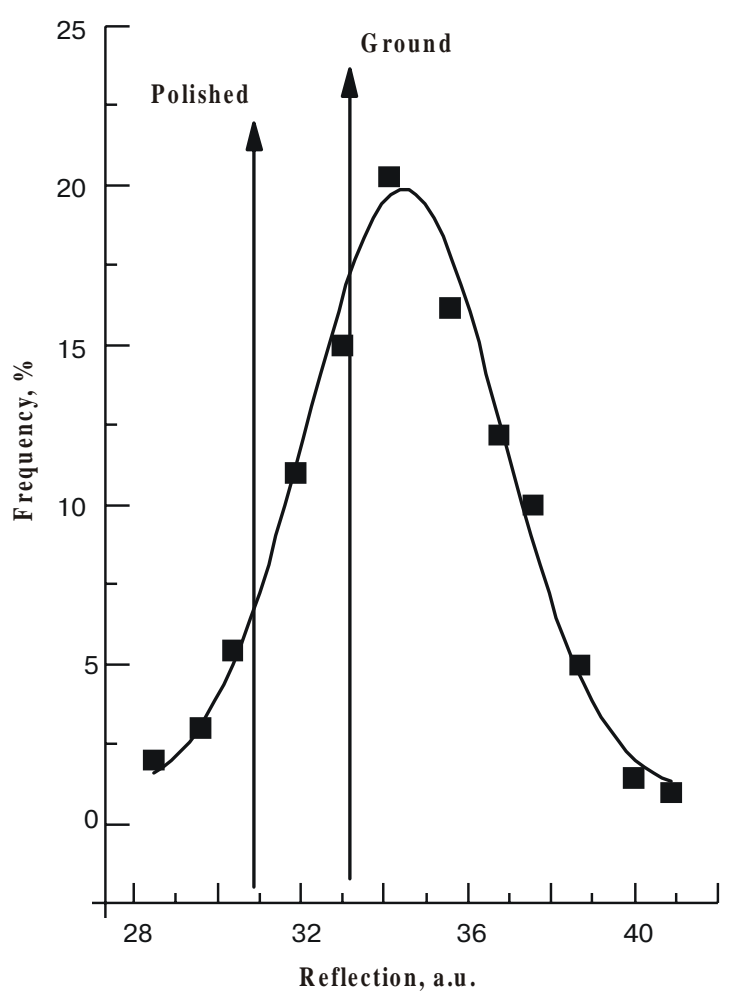

Fig. 5. The histogram of the $R$ parameter distribution for mc-Si sample and $R$ values for monocrystalline $\mathrm{Si}$ with polished (front side) and ground (rear side) surfaces (arrows). Points - experiment, curve - approximation by the Gauss function.

of a surface, and their distribution is featured by the Gauss function (full curve in Fig. 5). This effect is caused by different light scattering from crystal grains of different orientation, owing to selectivity of a chemical etching for the different planes.

The knowledge of $R$ parameter and its statistical distribution is important at development of texturization and antireflection technology optimization for mc-Si in the $\mathrm{SC}$ production route. The described method of $R$ measurements is attractive by virtue of its simplicity and expressness.

\section{Conclusions}

1. It is experimentally shown that at carrying out of single local measurements of a «solar» multicrystalline silicon parameters it is impossible to receive the correct values of these parameters due to distinction of their values in different (even close located) sites of the sample.

2 . The statistical distributions of grain sizes on a mcSi surface are explored. It is shown that it is possible to distinguish 4 groups of the crystal grain sizes; distribu- tion of the sizes in three groups (excluding extra large grains) is described by the Gauss functions.

3. The statistical distributions of the values of a diffusion length of minority non-equilibrium charge carriers and effective reflectivity of light from a mc-Si surface are explored. It is shown that these distributions are approximated by the Gauss functions.

4. The algorithm of mc-Si parameter determinations by the local measurements is described that permits to determine amount of measurements, necessary for deriving a parameter value distinguished from the mean one by the specified value of mean error. This procedure consists in measuring a statistical distribution of required parameter for 1-2 test samples of the batch, from which we gain a distribution dispersion and the values of the single measuring errors. The reaching of desirable precision is carried out by magnification of amount of measurements, according to the formula (3).

5. The described techniques of measurement and handling of the results obtained differ by a simplicity and expressness, and allow to optimize the processes of the mc-Si characterization, that is important for the development and manufacturing the mc-Si based solar cells.

\section{Acknowledgments}

The author would like to thank Prof. B. M. Romanjuk for helpful discussions and valuable remarks.

\section{References}

1. F. Ferrazza, Growth and Post Growth Processes of Multicrystalline Silicon for Photovoltaic Use // Solid State Phenomena, 51-52, pp. 449-460 (1996).

2. W. Warta, J. Sutter, B.F. Wagner, et al., Impact of Diffusion Length on the Performance of mc-Silicon Solar Cells // Proc. 2nd World Conf. on Photovoltaic Solar Energy Conversion. 6-10 July 1998, Vienna, Austria, pp. 1650-1653.

3. H.J. Moller, Multicrystalline Silicon for Solar Cells // Solid State Phenomena, 47-48, pp. 127-142 (1996).

4. A.M. Goodman, A Method for the Measurements of Short Minority Carrier Diffusion Lengths in Semiconductors // J. Appl. Phys., 32 (10), pp. 2550-2552 (1961).

5. Standard Test Methods for Minority Carrier Diffusion Length in Extrinsic Semiconductors by Measurements of SteadyState Surface Photovoltage. ASTM Designation: F391-96, published: April 1996 // Annual Book of ASTM Standards, v. 10.05 (Electronics II), pp. 150-158.

6. N.L. Dmitruk, Yu.V. Kryuchenko, V.G. Litovchenko et al., Diffusion Length Determination by the Surface Photovoltage Method // Phys. Status Solidi (a), 124 (1), pp. 183-190 (1991)

7. A.N. Zeidel. Errors of measurings of physical quantities, Science, Leningrad, 1974.

8. I.P. Lisovskii, V.G. Litovchenko, V.B. Lozinskii, et al., Properties of the Oxygen-Containing Silicon Single Crystals, Subjected to the Long-Time Thermal Treatments // Ukr. Fiz. Zhurn., 39 (1), pp. 68-73 (1994).

9. M. Shchigolev, Mathematical handling of observations, Science, Moscow, 1969. 